\title{
Facilitating Organizational Adoption of Sensor-Based Systems: Espoused Beliefs, Shared Assumptions and Perceived Values
}

\author{
Viktor Mähler \\ Umeå University \\ viktor.mahler@umu.se
}

\author{
Ulrika H. Westergren \\ Umeå University \\ ulrika.westergren@,umu.se
}

\begin{abstract}
The advent of sensor-based systems with their ability to collect, transmit and process context-aware data creates new opportunities for service delivery. We know from earlier research that there may be barriers to the adoption of new information technology (IT) within an organization. Sensor-based systems, with unprecedented potential for monitoring of products, people and processes are an interesting mix of potential and risk. Through the lens of organizational culture theory, we examine the question: Given the ambiguity and complexity of sensor-based systems, how does organizational culture influence perceptions of system value and purpose, and which factors determine the susceptibility of adoption among individual workers and teams? Our results suggest that the adoption of sensor-based systems is facilitated by 1) a basic comprehension of the system, its functionality, purpose and limitations; 2) a shared view of stakeholders' roles and responsibilities, and 3) a pronounced and tangible vision for value creation.
\end{abstract}

\section{Introduction}

Organizational adoption of IT has long been a central topic within the information systems' (IS) discipline. Ever since technology started being used in the workplace in the 1950s, scholars have been interested in studying why, how, and when IT is successfully adopted. A basic assumption has been that IT has an effect on the organization into which it is implemented and that the organization, in turn, has an effect on the technology and how it is used $[21,7]$.

A current technological phenomenon is the emergence of the Internet of Things (IoT) with a rapidly developing assortment of sensor-based systems that collect, transmit, and process data in real time. These systems are used to connect physical objects to the Internet and to generate, gather and analyze data in order to detect trends and anomalies, provide product and process information, and monitor and control specific contexts [28]. Utilizing the possibilities afforded by IoT involves mutual adaptation between work routines and technical tools - a socio-technical process that affects the entire organization.

Sensor-based systems have the potential to change the ways organizations do business and organize their work. The key is the data that is collected. For example, a product supplier with access to data from a product-in-use, may charge their customers based on usage rather than per unit delivered. A deeper understanding of a product in use can prevent costly unplanned stops and product failure and enable the service organization to adapt its business model and plan and deliver services more efficiently. Data also makes it possible for a supplier to efficiently monitor products and offer services after the point-of-sale and for a service provider to base service decisions on facts instead of educated guesses [29].

However, sensor-based systems and the data they generate also reveal information about people, from their whereabouts and movements, to their work pace and personalized routines. More importantly, they can do this simply by monitoring their environment and cross-analyzing data from different systems, without the worker actively engaging in the technology, by for example wearing a smart badge or logging in on a computer [4]. This "panoptic power" of IT has earlier been described by [33], who used it to denote an environment where you would not know if you were being surveilled, but you would be aware that the possibility always existed. By installing sensor-based systems, ordinary work environments have the potential to become embedded panopticons [17] and issues of privacy, security and control must therefore be a natural part of the discussion when it comes to the IoT [28].

The great potential that lies within the IoT when it comes to new ways of organizing work and delivering services, in combination with the unprecedented potential for simultaneous monitoring of products, people and processes, makes the adoption of sensorbased systems in an organizational context exceedingly 
interesting to study. In this paper we therefore ask: Given the ambiguity and complexity of sensor-based systems, how does organizational culture influence perceptions of system value and purpose, and which factors determine the susceptibility of adoption among individual workers and teams?

We follow two different firms, CleanCo - a private actor providing cleaning services to third parties, and MuniciClean - a municipal organization, providing cleaning and maintenance services within public facilities. At the time of the study, CleanCo had been using an implemented, externally developed, sensorbased system for a little over a year, in order to enhance their cleaning service delivery. MuniciClean, on the other hand, were about to begin the implementation of a similar, but internally developed, system within their organization. By comparing and contrasting experiences from two organizations with similar systems, but operating within different contexts, we are able to provide a rich picture of organizational adoption of sensor-based systems.

\section{Sensor-based systems and organizational adoption of technology}

It is widely recognized that as information is increasingly digitized and mobile devices accelerate in pervasiveness and processing power, an arena and architecture for innovation is opened up - one in which physical and digital components are combined [10, 32].

The Internet of Things (IoT) provides a complex environment where products are equipped with smart sensors and Internet connectivity [28]. These sensorbased systems are able to collect, process and transmit context-aware data thus through time and space opening up new arenas for service creation and business opportunities [18, 13]. The IoT is expected to grow exponentially in scope and is made possible through the development of cloud computing, miniaturization, smart sensors, and mobile technology [3].

There are many potential application areas for the IoT. As the real, digital, and virtual worlds converge, the IoT holds promise of smart cities, intelligent transportation solutions, vast energy savings, industrial and agricultural innovation and much more [28, 14]. The possibility of real time monitoring in combination with analysis of context-aware data opens up avenues for enhanced service delivery, improved product development and making informed choices [25]. Furthermore, one of the major opportunities for IoTenhanced business is the creation of business ecosystems where each participating actor contributes to the benefit of the whole group [22].
However, the omnipresence of technological solutions that continuously sense, monitor, and report contextual data also creates an environment where monitoring and surveillance pertains not only to products, but also people and processes. Jonsson [17] draws on Zuboff [33] and describes the potential panoptic power of remote monitoring systems. She shows how visibility and non-visibility of systems are closely related to the feeling of being monitored. Sensor-based systems, where sensors are embedded in objects, thus obscuring cues for surveillance, are therefore likely to evoke less negative feelings, than a pronounced, physical object, such as a surveillance camera mounted in an office corridor. This suggests that individual workers cannot oversee potential ethical dilemmas connected to the use of sensor-based systems, and that the responsibility for ethically sound use of the technology lies with both suppliers and upper management [17].

As more and more products are interconnected, there are thus a number of issues that must be addressed; both technological issues such as standards and interoperability of systems [28], human issues of privacy, security and control [17,], and organizational issues, such as adoption processes, value creation, and business model innovation [25]. Most of the IoT research to date, however, has focused on the technological advancements and not on the organizational aspects of the emerging IoT [30], and while a lot of optimism surrounds the concept, there is a need for more research that explores, and provides examples of, how sensor-based systems are adopted and diffused within organizations.

Earlier research has shown that organizational adoption of new technology is contingent upon many factors [1], including internal factors such as power structures and worker motivation and qualifications [5], technological attributes such as learnability and perceived usefulness [8], and organizational factors such as leadership, financial capability and organizational culture $[23,12]$. While few studies exist that specifically target organizational use of sensorbased-systems, previous research has suggested that employees who experience an organizational culture with strong human relations values report a higher level of readiness for change in adopting a new system $[6,16]$, and that adverse group-held values, could negatively affect the willingness to work- and promote working with a new system [11].

Organizational culture theory, introduced by E. H Schein [26] highlights assumptions, beliefs, shared values and norms within an organization. Sensor-based systems carry, on the one end, hopes of value creation and on the other, fears of excessive monitoring and breach of privacy. We therefore use this theoretical 
framework to highlight individual workers' conceptions of technology as a means to create an understanding for which factors determine the susceptibility of sensor-based system adoption among individual workers and teams. The next section gives a detailed overview of the framework and its main concepts.

\section{Organizational culture}

Organizational culture theory was first developed by Schein in the 1980s [26] and has since been applied in a variety of settings, including investigating the cultural aspect of knowledge management [2] and IT adoption [9], and is considered an accepted way of examining culture and values in the terms of IS [19]. The framework consists of three different 'levels', described as degrees of visibility in regards to a cultural phenomenon for an outside observer [27]. This framework aims to organize culture from that which is tangible to underlying assumptions that are notably more abstract. The three levels are divided into three categories; 1) Artifacts, 2) Espoused beliefs and values and 3) Basic underlying assumptions, in order of decreasing tangibility.

Artifacts represents a multitude of observable aspects that are considered tangible, including technology, group behavior and dynamic, and the emotional and physical environment of the employees. For the purpose of this study with its specific focus on technology adoption, we delimit the concept of artifacts to denote the digital artifact. This includes the sensor-based system itself and the expressed understandings and expectations that surround it from each of the participants.

Espoused beliefs and values entails beliefs, statements or values that may appear as official standpoints of an organization and used to create a sense of togetherness. They may also stem from a person perceived as a leader within a group. An espoused belief can initially be questioned or challenged but if it is perceived as "successful" among a group of people, it may evolve to become a shared assumption and viewed as the only viable solution to a general problem. This is a form of social validation, where joint experiences as a group validate beliefs and values, and shared assumptions serve to reduce uncertainty in vital areas of the functions pertaining to the group without any empirical merit.

Basic underlying assumptions are statements, beliefs and "know-how" that have worked sufficiently to be taken for granted as solutions to particular problems, even if they originally started off as simply a 'hunch' of how things ought to be. It can be beliefs or values that are so prevalent that they are viewed as facts or that any variation is a deviation from the norm or even inconceivable. This can manifest on both an individual-, group- or societal-level. Basic underlying assumptions may also be manifested through individuals or groups sharing subconscious ideas or values, coloring their perception of reality.

Together, these three concepts - Artifacts, Espoused beliefs and values, and Basic underlying assumptions - create an environment with certain norms, values and beliefs. By examining people's conceptions of technology from these three conceptual levels, we are provided with the necessary tools for understanding how organizational culture is created and realized, and thereby also given the means to investigate preconditions for technology adoption.

\section{Research methodology}

This research is based on a qualitative case study [20] focused on CleanCo and MuniciClean, two similar service providers, operating in different contexts. A majority of the data collection was done through semistructured interviews, where the strategies, thoughts and motivations behind the implementations were discussed, with a multitude of actors, ranging from an operative level, to middle management and up to a management-level. Through the interviews we could gain insight in regards to the motivations and rationale exhibited by the different actors in their respective roles [31].

\subsection{Research context}

The study is based on the case of two different organizations, CleanCo - a private actor providing cleaning services to third parties, and MuniciClean - a municipal organization, providing cleaning and maintenance services within public facilities. At the time of the study, CleanCo had been using an implemented, externally developed, sensor-based system to enhance their cleaning service delivery. MuniciClean, on the other hand, were about to begin the implementation of a similar, but internally developed, system within their organization.

The organizational structure within MunciClean consists of cleaners on the operative level reporting to a team-leader. The team-leaders then reports to their respective district manager, being the highest-level manager within that particular district.

In this particular case, the prototype development that MuniciClean was involved in featured the team leader for a pilot team of cleaners, two district managers, and a project manager, acting as managing 
director of the project - all based within the municipality. A third-party developer had been hired by the project manager, but at the time of our study, they had not yet been involved in any actual development. While the prototype developed for MuniciClean had not yet been implemented, CleanCo had been using their system for roughly a year at the time of the interviews.

CleanCo has a similar structure with cleaners, however the team leader worked $20-30 \%$ at an operative level, with the rest of the time being spent on administrative tasks, including the configuration and control of the graphical user interface for their sensorbased system, delegating tasks and similar details.

While the team leaders for CleanCo had managers within their own organization, they ultimately answered to the building manager of CorpHouse; the customer of CleanCo owner of the sensor-based system and the building and where cleaning was performed.

The system being used by CleanCo was developed by a third-party developer, BobSys, who had sold the system as a package deal to the building manager at CorpHouse, meaning that while CleanCo where using the system, they did not own it themselves.

While the two cases are mainly looking at CleanCo and MuniciClean, interviews were also performed with the developers of the system used by CleanCo, and also the building manager that contracted them in the first place. All firm names have been fictionalized in order to protect privacy.

\subsection{Data collection}

The snowball sampling was chosen as we were dealing with small population size featuring characteristics specific for this project [24], where we initially talked to the manager responsible for the project involving a sensor-based system for MuniciClean, and through the project manager established contact with the other key participants within the municipality - from district levels to the operative level of the cleaning-personnel themselves. In doing so we managed to cover all the municipal actors involved in the project and gain insights from everyone that was currently involved in the project.

Contact with CleanCo was established through the manager of CorpHouse, where CleanCo provided cleaning services, with the sensor-based system already installed upon accepting the contract. In doing lateral interviews with both MuniciClean, CleanCo, BobSys and CorpHouse, we argue that we have covered the actors necessary to understand the reasoning behind the adoption decisions at both MuniciClean and CleanCo.

The average interview lasted 45 minutes, with the longest interview lasting 170 minutes. A total of 16 interviews were performed and the interviews were all audio-recorded as well as transcribed.

\begin{tabular}{|l|l|l|}
\hline Organization & Person & Role of respondent \\
\hline \multirow{3}{*}{ MuniciClean } & $\mathrm{MC} 1$ & Project Manager \\
\cline { 2 - 3 } & $\mathrm{MC} 2$ & District Manager I \\
\cline { 2 - 3 } & $\mathrm{MC} 3$ & District Manager II \\
\hline $\begin{array}{l}\text { MuniciClean } \\
\text { (Continued.) }\end{array}$ & $\mathrm{MC} 4$ & Team Leader, Maintenance \\
\cline { 2 - 3 } CleanCo & $\mathrm{MC} 5$ & Cleaner \\
\hline \multirow{4}{*}{ BobSys } & $\mathrm{CC} 1$ & Team Leader, Maintenance \\
\cline { 2 - 3 } & $\mathrm{CC} 2$ & Team Leader, Super User \\
\cline { 2 - 3 } & $\mathrm{CC} 3$ & Cleaner I \\
\cline { 2 - 3 } & $\mathrm{CC} 4$ & Cleaner II \\
\hline & $\mathrm{BS} 1$ & Technical Manager \\
\cline { 2 - 3 } & $\mathrm{BS} 2$ & $\begin{array}{l}\text { Customer Relations / } \\
\text { Product Owner }\end{array}$ \\
\cline { 2 - 3 } & $\mathrm{BS} 3$ & Sales Representative \\
\hline \multirow{2}{*}{ CorpHouse } & $\mathrm{CH} 1$ & Building Manager \\
\hline
\end{tabular}

Table 1. Table detailing the interview respondents, their roles and the organization that they are in.

\subsection{Data analysis}

The data analysis was performed through the use of the organizational culture framework, where the interviews were read, and coded based on the theoretical categories of Artifacts, Espoused beliefs and Basic underlying assumptions, as described by Schein [27]. We searched for commonalities and discrepancies within the perception of the system and culture amongst the respondents, and compared them side-by-side, as can be seen within the result.

\section{Results}

The results section is structured according to the three theoretical concepts of Artifacts, Espoused beliefs and Underlying assumptions providing an overview of the respondents' thoughts and opinions on the sensor-based system.

\subsection{Artifacts}

The system used by CleanCo was purchased by CorpHouse from BobSys and already in place when Clean Co was contracted to provide cleaning services. It featured sensors being implemented in areas that required refills, such as soap, toilet paper and paper towels. It also featured a motion sensor that would measure the in and out passages from toilets and other areas that was deemed important by the building 
manager. The system was presented through a tablet interface, detailing different areas and their status which ranged from red (bad/empty/dirty), yellow (needs attention) to green (good/full/clean).

Both of the team leaders at CleanCo were invited to a presentation about system usage that was being held by the system supplier.

Since the system was already a finalized product, the presentation made by BobSys allowed for the team leaders to ask questions regarding system functionality, and in which ways it would fit into their work. It was described by $\mathrm{CC} 2$ as:

"The system is comparatively easy in the sense that; you arrive to an area and then you get it fairly well described [...] 'Here's a room', 'I have a header and under the header it tells me what to do here. [...] So it's a good tool to help you prioritize and especially to signal when it needs to be cleaned in various areas." CC2 (Team-leader, Super-User)

The team-leader CC1, with more operative duties, was asked to elaborate on the aspects, both negative and positive that they had experienced from the system:

"Routine-based it's better [using the system]. It becomes more effective when you already have sensors in... Well in most places. So you can see how many people [that are] going in or out [from the area]."CC1 (Team-leader, Maintenance)

While interviewing the cleaners, neither of them described any perceived negative aspects that they had noticed. CC2, however, stated that they had been noticing a stress aspect to the system:

"[...] it's just the stress aspect. That you never really know when you'll be automatically assigned to do another task. Apart from that I haven't heard that much regarding improvement or decrease." -CC2 (Team-leader, Super-User)

The building manager at CorpHouse, was asked to give his perception of the system and its reception:

"What we were afraid of was how the maintenance workers would perceive [the system]. If it was some kind of 'Big brother is watching' and those kinds of things, but it has... We used to have [previous company] and now we have CleanCo, and neither... neither of them has experienced any problems, from what I've heard. Instead it was only cool that they also got some new technical [gadgets]." -CH1 (Building Manager)
MuniciClean were busy developing a prototype system. The project team included all of the respondents within MuniciClean apart from MC5. A third-party developer had been included into the project but had not begun any phase of development, so all team members were internal to the organization. The purpose of the prototype, was to function as an indicator for the cleaning personnel, displaying the inand out passages from areas and rooms, and using a graphical user interface to show the cleaners where cleaning was necessary based on people passing:

"Number one *speaking from cleaners' view* is that 'The actual need for maintenance within an area is not known.', 'We go around cleaning whether it's necessary or not.'... *Speaking from own view* And that means that we have presumably inefficient resource utilization." - MC1 (Project Manager)

We asked the MuniciClean team leader to explain the thinking behind the development of the system; its role, functionality and limitations:

"Honestly I don't really know. I was kind of thrown into this where they said 'Hey, you're supposed to attend these meetings' and I said 'Uh-huh, what is this?', -MC4 (Team-Leader, Maintenance)

Both district managers had fairly similar viewpoints in regards to the rationale behind the prototype and MC3 summarized the expectations as:

"With these sensors you're supposed to read that 'Oh, in this conference room there hasn't been anyone -I don't have to go there' [...] - providing the cleaning where it's most required" -MC3 (District Manager II)

The cleaners that were affected by the prototype received their information from the team leader MC4. The cleaner that we interviewed had a similar understanding as the district managers regarding the functionality of the prototype, however when asked to speculate as to the reason behind the prototype they answered:

"Actually I have no idea... because.. eh.. [...] Well say that you have several floors and corridors that are long as hell, [then] it might improve... But I mean for us here it's not really like you'll kill yourself if you've walked through a corridor unnecessarily." -MC5 (Cleaner)

When asked about how the operative aspect of the prototype was viewed, the Project Manager stated that: 
"[...] So it's those things that we have been talking about; 'There's an economic incentive', we don't know what it looks like, but we think that we can ... better utilize the resources. Potentially you might be able to... utilize the resources differently [...] It could be that you formulate a new [cleaning] contract". -MC1 (Project Manager)

\subsection{Espoused beliefs}

The espoused beliefs were mostly expressed by the team leaders and managers, and, in the case of CleanCo, eventually evolved into shared assumptions. Two noteworthy aspects regarding the espoused values at CleanCo were the surveillance aspect of the system itself and the potential for stress. When we asked the Maintenance workers if they ever felt like there was a surveillance aspect to the system, the answers were all that they had not, with Maintenance Worker CC4 stating that:

"The thing is that you don't have the customer over your shoulder in the same way" -CC4 (Maintenance Worker II)

By using the system, dispensers would, for example, be re-filled in time, meaning the customer would be more satisfied and not perform as many manual check-ups as before. However, this perceived control function was in part replaced by system functionality, as the building manager, to some extent, used system data to monitor the overall cleaning being done, albeit not individual workers. While showcasing the system, and presenting a statistics page they commented:

"So it is here I'll... that I'll... on the level that I evaluate if they are doing a good job, or... we want as little critical time as possible. So I look at this when I'm about to discuss with their managers." -CH1 (Building Manager)

Where 'critical time' in this sense means time that an area or object has not been cleaned and remained in the 'red' status in the system for a prolonged time. While the espoused belief was that they did not want the workers to feel a big brother presence, there was an element of monitoring of the work being performed.

The only tangible negative aspect that was brought up during the interviews with the cleaners was the perceived stress aspect of at least one other cleaner at CleanCo, according to one of the team leaders. We interviewed the supplier about what the system meant for the cleaners, who stated:
"Well for the cleaners it means that they have a whole new kind of control over their area, it decreases their stress because they know where they should go and refill [material], they don't have to worry 'Where should I go first now?'." -BS2 (Product Owner / Customer Relations)

During one of the meetings between CleanCo and BobSys a functionality within the system that used to rate the cleanliness of an area was identified by one of the team leaders as a stress factor; as it could be interpreted as performing an assessment on how the previous cleaner had performed. This was no cause for alarm, according to the supplier, who simply stated that it was not the way to think about the system and continued their presentation without further discussion:

"And that's a way that you should not think, because the statistics we get is very anonymous, we can't see what time it is, at least not today." -BS2 (Product Owner / Customer Relations)

There were also two common espoused beliefs at MuniciClean, the first being that there existed an uncertainty as to the purpose of the system, and the other one was that the team leader had little to no technical knowledge, to the point where it was more of a funny thing to imply rather than a touchy subject, with the team leader jokingly stating that:

"[...] And I'm like 'I have no idea about any system. How things work.' So many things just go straight over my head and I'm like *puts face in hands* 'I don't understand anything', like 'Why am I sitting here?'." MC4 (Team-Leader, Maintenance)

This was further reaffirmed by both the cleaner within their group, describing how they had heard MC4 describe themselves, and one of the district managers describing $\mathrm{MC} 4 \mathrm{~s}$ technical knowledge as;

"Technology is not their thing. And now there's; 'here's a Tablet', [they] don't even know how to start it... [The Team-Leader] is young." -MC2 (District Manager I)

Apart from this aspect, what was being touched upon earlier was that there existed differing understanding on what the prototype was actually supposed to do and its purpose:

"I don't think the expectations are that we at the end of this project will have a concept for need-based cleaning - but we will have a material where we can 
[make requirements] for need-based cleaning." -MC1 (Project Manager)

This suggests that the development of the prototype system was interpreted as a pilot to test the viability of need-based cleaning, rather than a first step in rolling out a system of their own.

When the team leader was asked to elaborate on their view regarding the prototypes purpose, they stated their belief that:

"Well the municipality always wants to save, actually that's what it boils down to. And it's like 'Do we really need 11 employees here, considering the need?' we might go down to 8 [employees] so I think it could be a bit of that too." -MC4 (Team-Leader, Maintenance)

The district manager, disagreed, and provided a third perspective as to the purpose of the prototype:

"The purpose is absolutely not to reduce personnel; the purpose is to focus the cleaning where it is needed the most. [...] Preemptively cleaning, so that there isn't a room that is really messy an entire day [...] I think that will be perceived as better quality" -MC3 (District Manager II)

\subsection{Basic underlying assumptions}

At MuniciClean the underlying assumptions that were observed differed to those observed at CleanCo. CleanCo did not describe any major problems with either the system or the system supplier, however; MuniciClean, on the other hand, appeared to have readily identifiable underlying assumptions about the purpose of the system and about the municipality itself. When discussing the rationale for the hiring of CleanCo and the procurement of the system, the building manager for CorpHouse was overall pleased with both the system itself as well as the employees at CleanCo, stating:

"We had a lack of quality. So we have the same amount of personnel, but we have significantly better results." -CH1 (Building Manager)

When asked if whether or not the CleanCo cleaners had experienced any trouble with the system or employer, none stated that they had - and neither the cleaners nor the team leaders brought up any problems that they had encountered. One positive aspect according to the team leaders was the possibility to directly contact the system supplier BobSys in regards to any problems or suggested improvement of the system. CC2 described the co-operation as:
"They are very accommodating. [...] I have been able to provide feedback and so far it has seemed like they've been well received [...] I've been able to say 'Oh, can you do this?' and it's been done." -CC2 (Team-leader, Super-User)

The rationale behind the way BobSys introduced the system, through presentations, meetings, and in simplifying ways of feedback, was motivated as:

"What I've learned about the implementation is that you have to push this a lot - they have to be willing to change, and the managers have to... you can't just implement a system and hope it will solve itself, but you have to work with the system." - BS2 (Product Owner / Customer Relations)

Among the operative level at MuniciClean there instead existed an underlying feeling of unfairness within the municipality itself. This was mostly in relation to the way that the cleaners were treated by other colleagues within the municipality and their own perceived status. MC5 described the feelings regarding the system prototype as:

"[...] I do get really surprised; considering the fact that you [as a cleaner] are furthest down in anything imaginable, that they want to waste a bunch of tablets and this type of system on us... It feels like *laughs*... It feels... weird... actually, because I mean, we don't even get [free] coffee *laughs*" -MC5 (Maintenance Worker)

The view was shared by the Team-Leader as well, stating that:

"[...] and I came with ideas and like 'This way it would be... easiest for us to understand' but then they were like 'yeah, but this does not work'. [...] It's like you have different ways of thinking. [...] And then you're also small and young and [it's like] 'Aww, you work as a cleaner' pat on the head... a little pat on the head sometimes and you're like 'Mhmm..'.'-MC4 (Team-Leader, Maintenance)

While neither MC4 nor MC5 said that it was something that they had been explicitly told, there was a feeling of distrust towards what was considered the 'real' or 'hidden' reason for the prototype, and a feeling of something not making sense. The managers also took the perceived low status of cleaners for granted, with the project manager, when asked what they viewed as most exciting, expressed that: 
"I think this is the most interesting thing, because I think it's an area... well partly cleaning and maintenance, especially cleaning, is a low status job. It's always been a job where *mumbles embarrassed* 'Yeah, I work with cleaning', but that... in itself... they have no system. They're completely analogue." -MC1 (Project Manager)

\section{Discussion}

Through the lens of organizational culture theory, we've discerned three distinct themes related to the organizational adoption of sensor-based systems: 1) System comprehension, detailing individual workers' views on the system, its function, role and limitations, 2) The power of espoused beliefs and shared assumptions emphasizing collaboration within each of the organizations, and 3) Perceived value creation.

\subsection{System comprehension}

The workers of CleanCo had been introduced to a pre-made system that was presented to the teamleaders - providing them with a greater understanding of the system and its possibilities. The team leaders were also invested in the system itself, and perceived that it made their tasks easier to perform, and that the it did not make them feel like they were being watched, but rather the opposite - that it allowed them a degree of freedom as long as they performed their tasks.

The understanding had been achieved through meetings and presentations held by representatives from the system developer, where the team leaders at CleanCo participated. The purpose and functionality of the system was explained, all the while the developer also had a vested interest in bringing the team-leaders on board and expressing a positive outlook on the system.

MuniciClean on the other hand, did not have a system installed, they were busy developing it during the time of this study. The team leader was indeed participating in the development of the prototype system, but their own stated understanding of the functionality was lacking, and they did not feel that they could participate to the extent that they might have wanted. Furthermore, there existed an apparent mismatch between on the one hand, the project and district managers' and on the other hand the team leader's and cleaners' perception of what the system would be used for. While the managers saw the system as something that could provide information and improve working conditions, the cleaners suspected it would be used to cut down on personnel. Without addressing the issue of basic system comprehension and conveying the purpose of an implementation, the risk of an unsuccessful implementation increases [8]. As sensor-based systems operate without the worker actively engaging in them, some functionality remains hidden for the average worker. This can result in speculation of what the system can and cannot do and evoke a feeling of hidden agendas. In line with Jonsson's [17] recommendations, an important duty on the part of managers and system suppliers is therefore to "de-mystify" technology and make it more tangible and comprehensible for those affected by it.

\subsection{The power of espoused beliefs and shared assumptions}

Our analysis shows that the manifestation of espoused beliefs and shared assumptions [27] played a major role in workers' propensity to adopt sensorbased systems. At CleanCo the basic underlying assumption was that a co-operation between all three involved parties existed and was favorable to all actors. That in turn legitimized the espoused belief that the sensor-based system itself was beneficial to the organization.

CleanCo viewed co-operation as favorable since it gave them a sense of partnership with their customer and the ability to directly send input to the developer made them feel like their issues were being taken seriously. CorpHouse expressed that the system allowed them to experience a higher quality of service compared to before. The saw CleanCo's participation in regards to improvements and requirements, as a way to decrease their own administrative load, and used the generated statistics to assist in negotiations and business decisions with CleanCo. BobSys believed a favorable co-operation of all parties meant that their system would continue to be used by the building manager, and with the workers at CleanCo providing improvement for the system, it meant continuous user feedback for the development team. Hence, our results supports earlier research that claims that if cooperation is achieved and the team leaders support the system, there is a greater chance of an implementation succeeding $[27,16,6]$. It also highlights the issue of forming ecosystems to profit from the new technology, something that is noted as a salient feature of IoT solutions [22, 25], but not achieved in the case of MuniciClean.

At MuniciClean the basic underlying assumption was that the cleaners were the lowest ranking within the organization - a statement expressed both by the cleaners themselves and, although not maliciously, the managers spoke of the cleaning profession in general as a low status profession. We argue that this 
underlying assumption directly influenced the espoused belief that the prototype would not have any success, and that there was an ulterior motive behind the implementation.

The shared assumption that the team leader lacked technical knowledge and that they felt that technical terms or jargon went over their head, further reinforced the underlying assumption of inferiority, which of course could affect their desire to participate, and doubt that the prototype was for their benefit, leading to negative consequences in the implementation process [11, 15, 8]. This, in turn affected their perception of system value creation.

\subsection{Perceived value creation}

The third theme that emerged from our interviews was the diverse perceptions of system value creation. At CleanCo both individual workers and the three different organizations seemed to share a consensus about what their respective role was within the system, that they were dependent on one another in order to benefit, and that the value created for each organization was understood and implicitly agreed upon. Likewise, the system was already developed, with clear boundaries of what it could and could not do, presented by the developers to CleanCo, further making the vision of the value creation more tangible and understandable, potentially driving an increased chance of a successful implementation [7].

At MuniciClean, while co-operation was a goal, there did not seem to exist a shared consensus about the purpose of the prototype coupled with the aforementioned feeling of inferiority and suspicion among the participants that were going to use the prototype - risking a negative outcome $[8,16]$. The project manager, district managers, and team leader all had different perceptions about the value that was being created for each other and oneself. This might of course become clearer as the system is finalized and implemented, but failure to clearly express intended value creation and establish a mutual sense of purpose, affected perceptions of the system before it was even put to use and can create barriers for adoption as the process moves forward.

Another interesting result is that, there existed an aspect of stress amongst some of the workers at CleanCo and while the issue was acknowledged by the system supplier it was also readily dismissed. In fact, the system was described by the supplier as a way to decrease stress. This schism between the espoused value and actual value-in-use (or lack thereof) again ties into earlier findings [17] regarding the supplier's responsibility for ethically sound use of technology. It also shows the importance of having a pronounced and tangible vision for value creation, that is continually validated and evaluated.

\section{Conclusions and suggestions for future research}

This study takes a close look at the adoption of sensor-based systems within organizational contexts and answers the question: Given the ambiguity and complexity of sensor-based systems, how does organizational culture influence perceptions of system value and purpose, and which factors determine the susceptibility of adoption among individual workers and teams?

Through our analysis of two organizations, their sensor-based systems, and organizational cultures, we conclude that there are three prevalent aspects that emerge as important in facilitating sensor-based system adoption:

(1) A basic understanding of the system, its functionality, purpose and limitations

(2) A shared view of stakeholders' roles and responsibilities.

(3) A pronounced and tangible vision for value creation.

We argue that future research should look further into the organizational culture existing within both successful and unsuccessful implementations of sensor-based-systems, and the aspect of co-operation between different actors in the creation of an ecosystem surrounding sensor-based systems.

\section{References}

[1] Attewell, P. "Technology diffusion and organizational learning: The case of business computing." Organization science 3.1 (1992): 1-19.

[2] Alavi, M., Kayworth, T. R., and D. E. Leidner. "An empirical examination of the influence of organizational culture on knowledge management practices." Journal of management information systems 22.3 (2005): 191-224.

[3] Borgia, E. "The Internet of Things vision: Key features, applications and open issues", Computer Communications, Vol. 54, 2014, pp. 1-31.

[4] Brown, W. S. (1996). Technology, workplace privacy and personhood. Journal of Business Ethics, 15(11), 1237-1248.

[5] Bruque, S., \& Moyano, J. (2007). Organisational determinants of information technology adoption and implementation in SMEs: The case of family and cooperative firms. Technovation, 27(5), 241-253. 
[6] Burnes, Bernard, and Hakeem James. "Culture, cognitive dissonance and the management of change." International Journal of Operations \& Production Management 15.8 (1995): 14-33.

[7] Cabrera, Á., Cabrera, E. F., and S. Barajas. "The key role of organizational culture in a multi-system view of technology-driven change." International Journal of Information Management 21.3 (2001): 245-261.

[8] Calisir, F., and F. Calisir. "The relation of interface usability characteristics, perceived usefulness, and perceived ease of use to end-user satisfaction with enterprise resource planning (ERP) systems." Computers in human behavior 20.4 (2004): 505-515.

[9] Cooper, R. B. "The inertial impact of culture on IT implementation." Information \& Management 27.1 (1994): $17-31$

[10] Evans, D. S., Hagiu, A., and Schmalensee, R. 2006. Invisible engines: How software platforms drive innovation and transform industries. Cambridge, MA: MIT Press.

[11] Ferneley, E. H., and P. Sobreperez. "Resist, comply or workaround? An examination of different facets of user engagement with information systems." European Journal of Information Systems 15.4 (2006): 345-356.

[12] Fink, D. (1998). Guidelines for the successful adoption of information technology in small and medium enterprises. International journal of information management, 18(4), 243-253.

[13] Greengard, S. (2015). The Internet of Things. Cambridge, MA:MIT Press.

[14] Gubbi, J., Buyya, R., Marusic, S., and Palaniswami, M. 2013. "Internet of Things (IoT): A vision, architectural elements, and future directions," Future Generation Computer Systems, (29:7), pp. 1645-1660.

[15] Jackson, S. "Organizational culture and information systems adoption: A three-perspective approach." Information and Organization 21.2 (2011): 57-83

[16] Jones, R. A., N. L. Jimmieson, and A. Griffiths. "The impact of organizational culture and reshaping capabilities on change implementation success: The mediating role of readiness for change." Journal of Management Studies 42.2 (2005): 361-386.

[17] Jonsson, K. (2006). The embedded panopticon: visibility issues of remote diagnostics surveillance. Scandinavian Journal of Information Systems, 18(2), 3.

[18] Kortuem, G., Kawsar, F., Fitton, D., and Sundramoorthy, V. 2010. "Smart objects as building blocks for the internet of things," Internet Computing, IEEE, (14:1), pp. 44-51.
[19] Leidner, D. E., and T. Kayworth. "A review of culture in information systems research: Toward a theory of information technology culture conflict." MIS quarterly 30.2 (2006): 357-399.

[20] Mason, J. "Qualitative researching." London: Sage, 2nd edition, ISBN 07619.7428 (2002): 8.

[21] Orlikowski, W. J., and S. R. Barley. "Technology and institutions: What can research on information technology and research on organizations learn from each other?" MIS quarterly 25.2 (2001): 145-165

[22] Prince, K., Barrett, M., and Oborn, E. 2014. "Dialogical strategies for orchestrating strategic innovation networks: the case of the Internet of Things," Information and Organization, (24:2), pp. 106-127.

[23] Ramdani, B., Chevers, D., \& A. Williams, D. (2013). SMEs' adoption of enterprise applications: A technologyorganisation-environment model. Journal of Small Business and Enterprise Development, 20(4), 735-753.

[24] Ritchie, J., Lewis, J., Nicholls, C. M., \& Ormston, R. (Eds.). (2013). Qualitative research practice: A guide for social science students and researchers. Sage.

[25] Saarikko, T., U. H. Westergren, and T. Blomquist, "The Internet of Things: Are you ready for what's coming?", Business Horizons, Vol. 60, No. 5, 2017, pp. 667-676.

[26] Schein, E. H. Organizational Culture. WP 2088-88. Sloan School of Management Working Papers, Massachussets Institute of Technology, 1988

[27] Schein, E. H. Organizational culture and leadership. Vol. 2. John Wiley \& Sons, 2010.

[28] Vermesan, O., and Friess, P. (Eds.). 2013. Internet of things: converging technologies for smart environments and integrated ecosystems. Aalborg:River Publishers.

[29]Westergren, U. H., T. Saarikko, and T. Blomquist, "Initiating the Internet of Things: Early Adopters' Expectations for Changing Business Practices and Implications for Working Life", in The Internet of People, Things and Services, C. Simmers and M. Anandarajan, Eds., Routledge, London, 2018, pp. 111-131.

[30] Whitmore, A., Agarwal, A. and L. Da Xu, 2015. "The Internet of Things - A survey of topics and trends", Information Systems Frontiers, (17:2), pp. 261-274

[31] Yin, R. K. 2009. Case Study Research: Design and Methods, Thousand Oaks, CA: Sage Publications.

[32] Yoo, Y., Boland, R. J., Jr., Lyytinen, K., and Majchrzak, A. 2012. "Organizing for innovation in the digitized world,"Organization Science, (23:5), pp. 1398-1408

[33] Zuboff, S., (1988). In the age of the smart machine: The future of work and power, Basic Books, New York. 